\title{
Lesion in a Basis Function Model of Parietal Cortex: Comparison with Hemineglect
}

\section{A. POUGET ${ }^{\mathfrak{l}}$ and T. J. SEJNOWSKI ${ }^{2}$}

${ }^{1}$ Institute for Cognitive and Computational Sciences, Georgetown University, Washington D.C., USA

${ }^{2}$ Howard Hughes Medical Institute, The Salk Institute for Biological Studies, La Jolla, USA and Department of Biology University of California, San Diego, La Jolla, USA

According to current theories of spatial representation, the positions of objects are represented in multiple processing systems throughout the brain, each system specialized for a particular sensorimotor transformation and using its own frame of reference (Stein 1992; Goldberg et al. 1990). The lateral intraparietal area (LIP), for example, appears to encode the locations of objects in oculocentric coordinates, presumably for the control of saccadic eye movements (Colby et al. 1995). The ventral intraparietal cortex (VIP; Colby and Duhamel 1993) and the premotor cortex (Fogassi et al. 1992; Graziano et al. 1994), on the other hand, seem to use head-centered coordinates and might be involved in the control of hand movements toward the face.

This modular theory of spatial representations is not fully consistent with the behavior of patients with parietal or frontal lesions. Such lesions cause hemineglect, a syndrome characterized by a lack of response to sensory stimuli appearing in the hemispace contralateral to the lesion (Heilman et al. 1985). According to the modular view, the deficit should depend on behavior (e.g., oculocentric for eye movements, head-centered for reaching). However, experimental and clinical studies show a more complex pattern. Instead, neglect affects multiple frames of reference simultaneously, and to a first approximation, independently of the task.

This point is particularly clear in an experiment by Karnath et al. (1993; Fig. 1A). Subjects were asked to identify a stimulus that can appear on either side of the fixation point. In order to test whether the position of the stimuli with respect to the body affects performance, two conditions were tested: a control condition with head straight ahead (C1) and a second condition with head rotated $15^{\circ}$ on the right (where right is defined with respect to the trunk) or, equivalently, with the trunk rotated $15^{\circ}$ on the left (where left is defined with respect to the head) (see Fig. 1A, C2). In C2, both stimuli occurred further to the right of the trunk than in $\mathrm{Cl}$, though at the same location with respect to the head and retina. Moreover, the trunk-centered position of the left stimulus in $\mathrm{C} 2$ was the same as the trunkcentered position of the right stimulus in $\mathrm{Cl}$. 
A

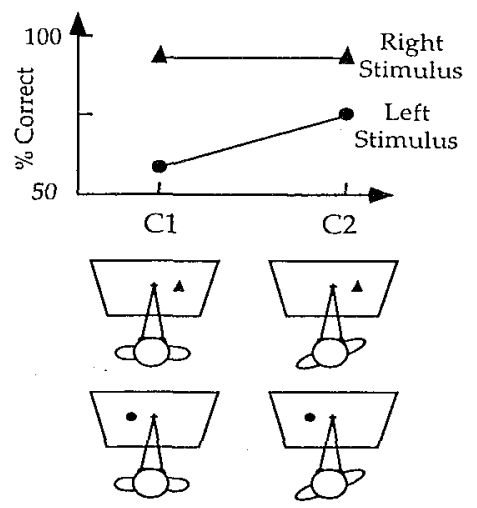

B

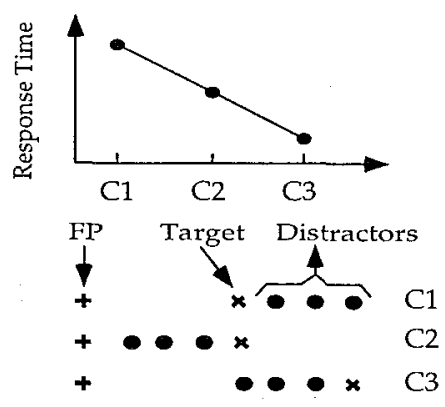

Fig. 1. A Percentage of correct identification in Karnath et al. experiment (1993). In condition $1(C 1)$, subjects were seated with eyes, head, and trunk lined up whereas in condition $2(C 2)$ the trunk was rotated by $15^{\circ}$ to the left. The overall pattern of performance is not consistent with pure retinal or pure head-centered neglect and suggests a deficit affecting a mixture of these two frames of reference. B Response times for Arguin and Bub (1993) experiment for the three experimental conditions illustrated below the graph ( $F P$, fixation point). The decrease from condition $1(C I)$ to condition $2(C 2)$ is consistent with object centered neglect, i.e., subjects are faster when the target is on the right of the distractors then when it is on the left, even though the retinal position of the target is the same. The further decrease in reaction time in condition $3(C 3)$ shows that the deficit is also retinotopic

As expected, subjects with right parietal lesions performed better on the right stimulus in the control condition (C1), a result consistent with both retinotopic and trunk-centered neglect. To distinguish between the two frames of reference, performance should be compared across conditions.

If the deficit is purely retinocentric, the results should be identical in both conditions since the retinotopic locations of the stimuli do not vary. On the other hand, if the deficit is purely trunk-centered, the performance on the left stimulus should improve when the head is turned right since the stimulus now appears further toward the right of the trunk-centered hemispace. Furthermore, performance on the right stimulus in the control condition should be the same as performance on the left stimulus in the rotated condition since they share the same trunk-centered position in both cases.

Neither of these hypotheses can fully account for the data. As expected from retinotopic neglect, subjects always performed better on the right stimulus in both conditions. However, performance on the left stimulus improved when the head was turned right (C2), though not sufficiently to match the level of performance on the right stimulus in the control condition (C1, Fig. 1A). Therefore, these results suggest a retinotopic neglect modulated by trunk-centered factors. 
In addition, Karnath et al. (1991) tested patients on a similar experiment in which subjects were asked to generate a saccade toward the target. The analysis of reaction time revealed the same type of results as the one found in the identification task, thereby demonstrating that the spatial deficit is, to a first approximation, independent of the task. Several other experiments have found that neglect affects a mixture of frames of reference in a variety of tasks (Ladavas 1987; Ladavas et al. 1989; Calvanio et al. 1987; Farah et al. 1990; Bisiach et al. 1985; Behrmann and Moscovitch 1994).

An experiment by Arguin and Bub (1993) suggests that neglect can be objectcentered as well. As shown in Fig. 1B, they found that reaction times were faster when a target (cross " $x$ " in Fig. 1B) appeared on the right of a set of distractors (C2) instead of on the left side (C1), even though the target is at the same retinotopic location in both conditions. Interestingly, moving the target further to the right led to even faster reaction times (C3), showing that hemineglect is not only object-centered but retinotopic as well in this task. Several other experiments have led to similar conclusions (Bisiach et al. 1979; Driver and Halligan 1991; Driver et al. 1994; Halligan and Marshall 1994; Husain 1995).

These results strongly support the existence of spatial representations using multiple frames of reference simultaneously shared by several behaviors. We recently developed a theory with these properties (Pouget and Sejnowski 1995, in press); we examine here whether a simulated lesion leads to a deficit similar to hemineglect. Our theory posits that parietal neurons compute basis functions (BFs) of sensory signals, such as visual or auditory inputs, and posture signals, such as eye or head position. The resulting representation, which we called a basis function map, can be used for performing nonlinear transformations of the sensory inputs - the type of transformations required for sensorimotor coordination.

The basis function hypothesis is briefly summarized in the first section of this chapter. In the second section, we describe the network architecture and the various methods used to assess the network performance in behavioral tests. In the third section, we compare the behavior of parietal patients with the network after a unilateral lesion of the basis function representation.

\section{Basis Function Representation}

The receptive field of most parietal cells is fixed on the retina, as for V1 neurons. The amplitude of their response to a light, however, is modulated by eye position (Andersen et al. 1985): typically, the response to a visual stimulus in the center of the receptive field increases monotonically as the eye moves along a particular direction in space, specific to each cell.

We have shown in a previous study that these response properties are consistent with the hypothesis that parietal neurons compute basis functions of their inputs 
A

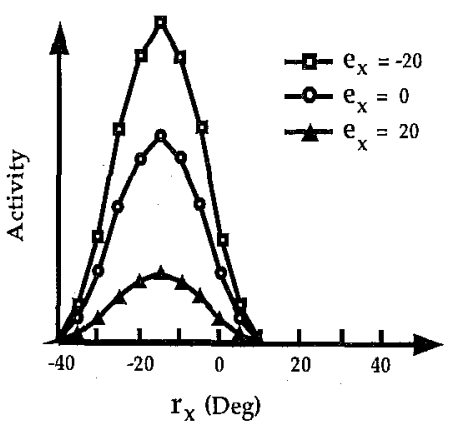

B

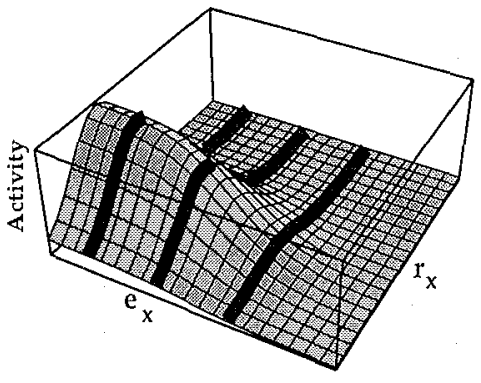

Fig. 2. A Idealization of a retinotopic visual receptive field of a typical parietal neuron for three different gaze angles $\left(e_{x}\right)$. Note that eye position modulates the amplitude of the response but does not affect the retinotopic position of the receptive field (adapted from Andersen et al. 1985). B 3D plot showing the response function of an idealized parietal neuron for all possible eye and retinotopic positions, $e_{x}$ and $r_{x}$. The plot in $A$ was obtained by mapping the visual receptive field of this idealized parietal neuron for three different eye positions as indicated by the bold lines

(Pouget and Sejnowski 1995, in press). Their response can be described as the product of a gaussian function of retinal location multiplied by a sigmoid function of eye position (Fig. 2B). Sets of both Gaussians and sigmoids are basis functions, and the set of all products of these two basis functions also forms basis functions over the joint space.

A set of basis functions has the property that any nonlinear function can be approximated by a linear combination of the basis functions. Therefore, basis functions reduce the computation of nonlinear mappings to a linear transformation - a simpler computation. Most sensorimotor transformations are nonlinear mappings of the sensory and posture signals into motor coordinates; hence, given a set of basis functions, the motor command can be obtained by a linear combination of these functions. Basis functions are precisely what parietal neurons appear to compute. This formalization entails that the parietal cortex recodes the sensory inputs in a format that facilitates the computation of motor commands. This perspective is consistent with the Goodale and Milner suggestion that the dorsal pathway mediates object manipulation (the "How" pathway), as opposed to simply localizing objects as Mishkin et al. previously suggested (the "Where" pathway) (Goodale and Milner 1990; Mishkin et al. 1983).

It is important to realize that not all models of parietal cells have the properties of simplifying the computation of nonlinear motor commands. For example, Goodman and Andersen (1990) as well as Mazzoni and Andersen (1995) have proposed that parietal cells simply add the retinal and eye position signals. The output of this linear model does not reduce the computation of motor commands to linear combinations because linear units cannot provide a basis set. By contrast, 
the hidden units of the Zipser and Andersen model (1988) have response properties closer to the basis function units, and the basis function hypothesis can be seen as a formalization of this previous model (for a detailed discussion see Pouget and Sejnowski, in press).

One particularly interesting property of basis functions is the fact that they represent the positions of objects in multiple frames of reference simultaneously. Thus, one can recover simultaneously the position of an object in retinotopic and head-centered coordinates from the response of a group of basis function units similar to the one shown in Fig. 2B (Pouget and Sejnowski 1995, in press). As shown in the next section, this property allows the same set of units to be used to perform multiple spatial transformations in parallel.

This approach can be readily extended to other sensory and posture signals and to other parts of the brain where similar gain modulations have been reported (Trotter et al. 1992; Field and Olson 1994; Boussaoud et al. 1993; Bremmer and Hoffmann 1993; Brotchie et al. 1995). When generalized to other posture signals such as vestibular inputs of head position, the resulting representation encodes simultaneously the retinal, head-centered, body-centered, and world centered coordinates of objects.

The study presented here explores the effects of a lesion in a spatial representation using basis functions. It is an attempt to bridge the gap between our current understanding of spatial representations at the neurophysiological and neuropschological levels.

\section{Model Organization}

The model contains two distinct parts: a network for performing sensorimotor transformations and a selection mechanism. The selection mechanism is used when there is more than one object present in the visual field at the same time.

\section{Network Architecture}

We implemented a network using basis function units in the intermediate layer to perform a transformation from a visual retinotopic map to two motor maps in head-centered and oculocentric coordinates respectively (Fig. 3). The input contains a retinotopic visual map analogous to the one found in the early stages of visual processing and a set of units encoding eye position, similar to the neurons found in the intralaminar nucleus of the thalamus (Schlag-Rey and Schlag 1984). These input units project to a set of intermediate units that contribute to both output transformations. Each intermediate unit computes a Gaussian of the retinal location of the object, $r_{x}$, multiplied by a sigmoid of eye position, $e_{x}$ : 


$$
o_{i j}=\frac{e^{-\frac{\left(r_{x}-r_{x i}\right)^{2}}{2 \sigma^{2}}}}{1+e^{-\beta\left(e_{x}-e_{x j}\right)}}
$$

We consider horizontal positions only because the vertical axis is irrelevant for hemineglect. These units are organized in two two-dimensional maps covering all possible combinations of retinal and eye position selectivities. The only difference between the two maps is the sign of the parameter $\beta$ which controls whether the units increase or decrease activity with eye position. $\beta$ was set to $8^{\circ}$ for one map and $-8^{\circ}$ for the other map. The indices $(i, j)$ refer to the position of the units on the maps. Each location is characterized by a position for the peak of the retinal receptive field, $r_{x i}$, and the midpoint of the sigmoid of eye position, $e_{x j}$. These quantities are systematically varied along the two dimensions of the maps in such a way that in the upper right corner $r_{x i}$ and $e_{x j}$ correspond to right retinal and right eye positions whereas in the lower left they correspond to left retinal and left eye positions.

As emphasized previously, this type of response function is consistent with the responses of single parietal neurons found in area 7a. The resulting population of units forms basis function maps encoding the locations of objects in head-centered and retinotopic coordinates simultaneously.
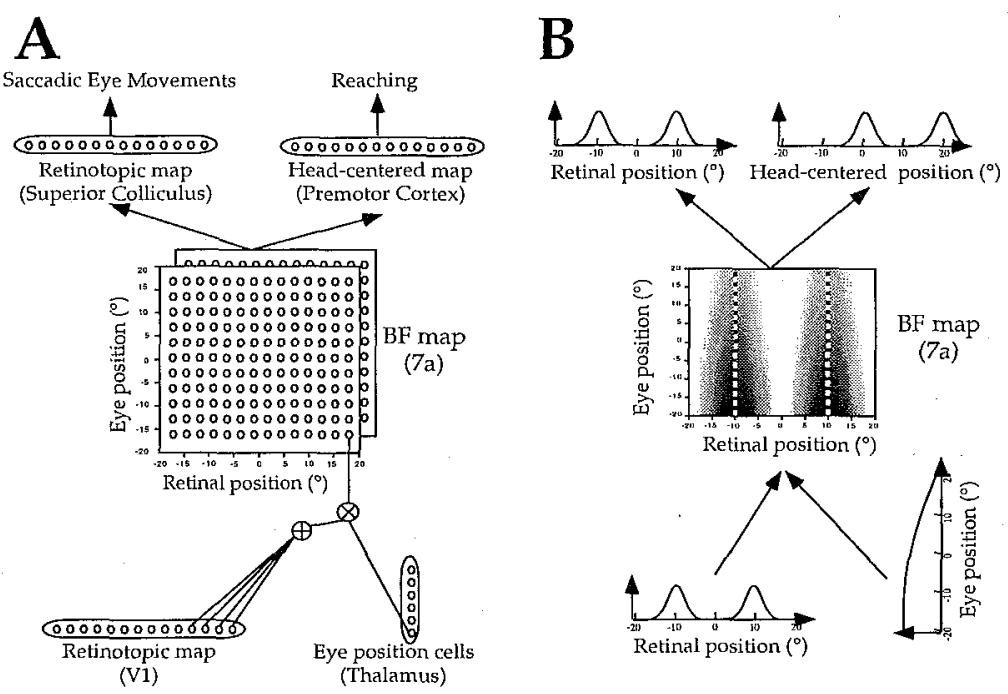

Fig. 3. A Network architecture. Each unit in the intermediate layers is a basis function unit with a gaussian retinal receptive field modulated by a sigmoid function of eye position. This type of modulation is characteristic of the response of parietal neurons. B Pattern of activity for two visual stimuli presented at $+10^{\circ}$ and $10^{\circ}$ on the retina with the eye pointing at $+10^{\circ}$ 
The activities of the units in the output maps are computed by a simple linear combination of the BF unit activities. Appropriate values of the weights were found by using linear regression to achieve the least mean square error.

This architecture mimics the pattern of projections of the parietal area $7 \mathrm{a}$, which projects to both the superior colliculus and the premotor cortex (via the ventral parietal area VIP; Andersen et al. 1990; Colby and Duhamel 1993), where neurons have retinotopic and head-centered visual receptive fields respectively. Figure 3B shows a typical pattern of activity in the network when two stimuli are presented simultaneously while the eye is fixated $10^{\circ}$ toward the right (only the BF map with positive $\beta=+8^{\circ}$ is shown).

\section{Hemispheric Biases and Lesion Model}

Neurophysiological data indicate that although the parietal cortices in both hemispheres contain neurons with all possible combinations of retinal and eye position selectivities, most cells tend to have their retinal receptive field on the contralateral side (Andersen et al. 1990). Whether a similar contralateral bias exist for the eye position in the parietal cortex remains to be determined although several authors have reported such bias for eye position selectivities in other parts of the brain (Schlag-Rey and Schlag 1984; Galletti and Battaglini 1989; van Opstal et al. 1995).

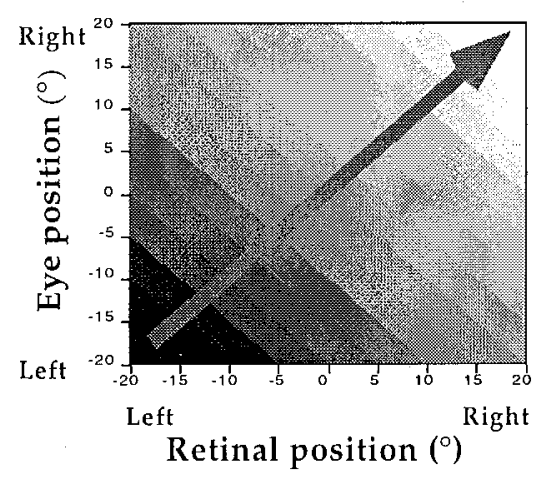

Left Map

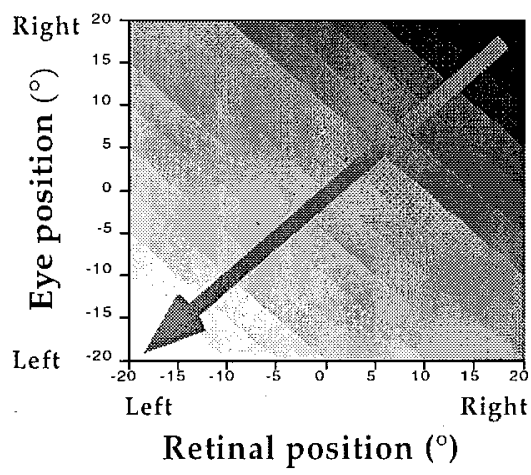

Right Map

Fig. 4. Neuronal gradients in left and right basis function maps for which the parameter $\beta$ is positive, i.e, the units increase activity with eye position. The right map contains more neurons for left retinal and left eye positions while the left map has the opposite gradient 
In the model, we divide the two BF maps into two sets of two maps, one set for each hemisphere (again the two maps in each hemisphere correspond to two possible values for the parameter $\beta$ ). Units are distributed across each hemisphere to create neuronal gradients. These neuronal gradients induce contralateral activity gradients such that there is more activity overall in the left maps than in the right maps when an object appears on the right of the retina and the eyes are turned to the right, with the opposite being true in the right maps.

Several types of neuronal gradients can lead to such activity gradients. The gradients we use for the simulations presented here affect only the maps with positive $\beta$, i.e, maps with units whose activity increases as the eyes turn right. In both the right and left map, the number of units for a given pair of $\left(r_{x i}, e_{x i}\right)$ increases for contralateral values of eye and retinal location as indicated in Fig. 4 which is consistent with the experimental observation that hemispheres overrepresent contralateral positions.

To model a right parietal lesion, we removed the right parietal maps and studied the network behavior with the left maps alone. The effect of the lesion is therefore to induce a neuronal gradient such that there is more activity in the network for right retinal and right eye positions.

We found that the exact profile of the neuronal gradient across the basis function maps did not matter as long as it induces a monotonically increasing activity gradient as objects are moved further to the right of the retina and the eyes fixate further to the right. The results presented in this chapter were obtained with linear neuronal gradients.

\section{Selection Model}

We adapted a selection mechanism from Burgess (1995) which itself was inspired by the visual search theory of Treisman and Gelade (1980) and the saliency map mechanism proposed by Koch and Ullman (1985). It was used to model the behavior of patients when presented with several stimuli simultaneously and it operates on what we call the saliency value associated with each stimulus.

The simultaneous presentation of stimuli induces multiple hills of activity in the network (see for instance the pattern of activity shown in Fig. 1B for two visual stimuli). We defined the stimulus saliency, $s_{i}$, as being the sum of the activities of all the basis function units whose receptive field is centered exactly on the retinal position of the stimulus (it is the sum of activities along the dotted line shown on the basis function map in Fig. 3B). The index $i$ varies from 1 to $n$, with $n$ as the number of stimuli in view at a given time. This method is mathematically equivalent to looking at the profile of activity in the superior colliculus output map and defining the saliency of the stimulus as the peak value of activity. Consequently, one need only consider the profiles of activity in the colliculus 
output map to determine the network behavior. Qualitatively similar values could also be obtained by looking at the profile of activation in the head-centered map.

At the first time step, the stimulus with the highest saliency is selected by winner-take-all, and its corresponding saliency is set to zero to implement inhibition of return. At the next time step, the second highest stimuli is selected, and inhibited, while the previously selected item is allowed to recover slowly. These operations are repeated for the duration of the trial. This procedure ensures that the most salient items are not selected twice in a row, but because of the recovery process, the stimuli with the highest saliencies might be selected again if displayed long enough.

This mechanism is such that the probability of selecting an item is proportional to two factors: the absolute saliency associated with the item and the saliency relative to the ones of competing items.

\section{Evaluating Network Performance}

We used this model to simulate several experiments in which patient performance was evaluated according to reaction time or percent of correct response.

In reaction time experiments, we assumed that processing involves two sequential steps: target selection and target processing. Target selection time was assumed to be proportional to the number of iterations, $n$, required by the selection network to select the stimulus using the mechanism described above. Each iteration was arbitrarily chosen to be $50 \mathrm{~ms}$ long. This duration matters only when more than one stimulus is present, so that distractors could delay the detection of the target by winning the competition.

The time (RT) for target processing (that is to say, target recognition, target naming, etc.) was assumed to be inversely proportional to stimulus saliency, $s_{i}$.

$$
R T=100+50 n+\frac{500}{1000 s_{i}} .
$$

We determined the percentage of correct responses to a stimulus to be a sigmoid of the stimulus saliency:

$$
p=\frac{0.5}{\left(1+\exp \left(-\left(s_{i}-s_{0}\right) / t\right)\right.}+0.5
$$

where $s_{o}$ and $t_{o}$ are constants.

This is a standard method in signal detection theory when assuming gaussian noise of equal variance for signal and noise (Green and Swets 1966). This is equivalent to assuming that the rate of correct detection (hit rate) is the integral of the probability distribution of the signal from the decision threshold to infinity. 
In line bisection experiments, subjects are asked to judge the midpoint of a line segment. Our network estimated the midpoint of a line, $\vec{m}$, by computing the center of mass of the activity induced by the line in the BF map.

$$
\vec{m}=\frac{\sum_{\text {all units }} a_{i} r_{x i}}{\sum_{\text {all units }} a_{i}}
$$

where $r_{x i}$ is the retinal position of the peak of the visual receptive field of unit $i$.

\section{Results}

All the results concern the lesioned model only, i.e., the model in which the right $\mathrm{BF}$ maps have been removed.

\section{Line Cancellation}

We first tested the network on the line cancellation test, a test in which patients are asked to cross out short line segments uniformly spread over a page. To simulate this test, we presented the display shown in Fig. 5A and ran the selection mechanism to determine which lines get selected by the network. As illustrated in Fig. 5A, the network crossed out only the lines located in the right half of the display, just as left neglect patients do in the same task (Heilman et al. 1985). The rightward gradient introduced by the lesion makes the right lines more salient than the left lines. As a result, the rightmost lines always win the competition, preventing the network from selecting the left lines.

We computed the probability that the line was crossed out as a function of its position in the display, where position is defined with respect to the frame of the display (Fig. 5A). We found that there is a sharp jump in the probability function such that lines on the right of this break have a probability near 1 of being selected whereas lines on the left of the break have a probability near zero (Fig. 5B).

The sharp jump in the probability of selection stands in contrast to the smooth and monotonic profile of the neuronal gradient. Whereas the sharp boundary in the pattern of line crossing may suggest that the model "sees" only one half of the display, the linear profile of the neuronal gradient shows that this is not the case. The sharp jump is mostly the consequence of the dynamics of the selection process: because right bars are associated with higher saliencies, they consistently win the competition to the detriment of left bars. Consequently, the network starts by selecting the bar the furthest on the right and due to inhibition of return moves its way toward the left. Eventually, however, previously inhibited items recover 
A

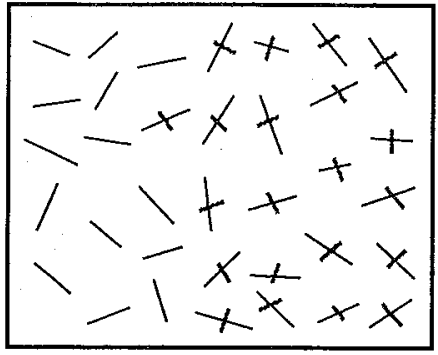

C

\section{B}

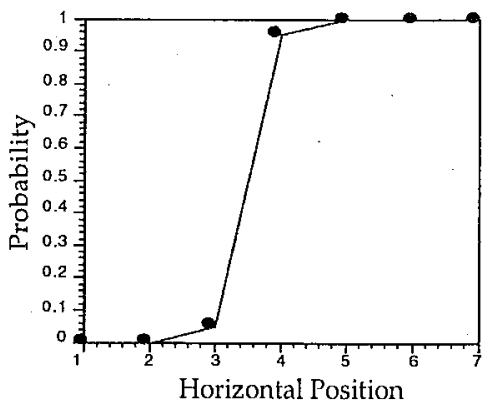

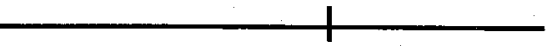

Fig. 5. A Network behavior in line cancellation task. As with right parietal patients, the network fails to cross out the line segments on the left of the page. B Probability of crossing a line as a function of its horizontal position in the display. The network behaves as if it had no representation of the left side of the display, i.e, as if the neuronal gradient introduced by the lesion were a step function. The gradient however is smooth, and the sudden change in behavior in the middle of the display is the result of the dynamics of the selection mechanism. C Network behavior in line bisection task. The midpoint is estimated too far to the right due to the overrepresentation of the right side of space

and win the competition again, preventing the network from selecting the leftmost bars. The point at which the network stops selecting bars toward the left depends on the exact recovery rate and the total number of items displayed.

The pattern of line crossing by the network is not the result of a deficiency in the selection mechanism. It is the result of a selection mechanism operating on a lesioned spatial representation. The network had trouble detecting stimuli on the left side of space not because it was unable to orient toward that side of space - it would orient to the left if only one stimulus were presented in the left hemifield but because the bias in the representation favored the rightmost bars in the competition.

\section{Line Bisection}

In the line bisection task, the network estimated the line midpoint to be slightly to the right of the actual midpoint (Fig. 5C) as reported in patients with left neglect (Heilman et al. 1985). In contrast, the performance of an intact network was perfect (not shown). 
The error was not because the lesioned network did not "see" the left side of the line. On the contrary, the whole line was represented in the lesioned network but due to the neuronal gradient, more neurons respond to the right side of the line than the left side. As a result, the center of mass calculation used to estimate the middle of the line leads to a rightward error.

Therefore, as assessed by the line cancellation and line bisection tests, a lesioned network exhibited a behavior consistent with the neglect syndrome observed in humans following unilateral parietal lesions.

\section{Mixture of Frames of Reference}

Next, we sought to determine the frame of reference of neglect in the model. Since Karnath et al. (1993) manipulated head position, we simulated their experiment by using a BF map integrating visual inputs with head position, rather than eye position. We show in Fig. 6B the pattern of activity obtained in the retinotopic output layer of the network in the various experimental conditions. In both conditions, head straight ahead (dotted lines) or turned on the side (solid lines), the right stimulus is associated with more activity than the left stimulus. This is the consequence of the larger number of cells in the basis function map for

A

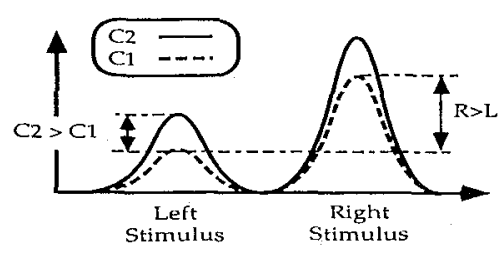

B
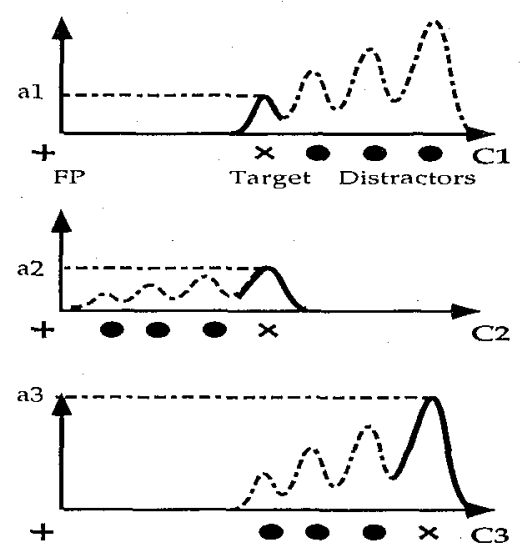

Fig. 6 A, B. Activity patterns in the retinotopic output layer when simulating the experiments by A Karnath et al. (1993) and B Arguin and Bub (1993). A Performance on the left stimulus improves from condition $1(\mathrm{Cl})$ to condition $2(\mathrm{C} 2)$ because the stimulus saliency increases across conditions. B Reaction time between conditions 1 and 2 decreases due to the change in the relative saliency of the target with respect to the distractors, even though the absolute saliency of the target is the same in these two conditions $(a I=a 2)$. FP, Fixation point; $C 3$, condition 3 
rightward position. In addition, the activity for the left stimulus increases when the head is turned to the right. This effect is related to the larger number of cells in the basis function maps tuned to right head positions.

Since network performance is proportional to activity strength, the overall pattern of performance was found to be similar to what has been reported in human patients (Fig. 1A): the right stimulus was better processed than the left stimulus, and performance on the left stimulus increases when the head is rotated toward the right, although not sufficiently to match the peformance on the right stimulus in condition 1. Therefore, as in humans, neglect in the model is neither retinocentric nor trunk-centered alone but both at the same time.

Similar principles can be used to account for the behavior of patients in many other experiments dealing with frames of reference (Ladavas 1987; Ladavas et al. 1989; Calvanio et al. 1987; Farah et al. 1990; Bisiach et al. 1985; Behrmann and Moscovitch 1994).

\section{Object-Centered Effect}

The network reaction times in simulations of the Arguin and Bub (1993) experiments followed the same trends reported in human patients (Fig. 1B). Figure $6 \mathrm{~B}$ illustrates the patterns of activity in the retinotopic output layer of the network for the three conditions in those experiments. Although the absolute levels of activity associated with the target (solid lines) in conditions 1 and 2 are the same, the activity of the distractors (dotted lines) differed in the two conditions. In condition 1, they had relatively higher activity and thereby strongly delayed the detection of the target by the selection mechanism. In condition 2, the distractors were less active than the target and did not delay target processing as much as they did in condition 1 . The reaction time decreased even more in condition 3 because the absolute activity associated with the target was higher. Therefore, the network exhibited retinocentric and object-centered neglect, with the same pattern observed in parietal patients (Arguin and Bub 1993).

The object-centered effect might not have been expected since there was no explicit object-centered representation in the model. This result demonstrates that object-based neglect does not necessarily imply that an explicit object-based. representation has been lesioned in neglect patients. The form of neglect found in the Arguin and Bub (1993) experiment could be a consequence of relative neglect since the apparent object-based effect could be explained by the relative saliency of the subparts of the object.

Other results in neglect can be explained with the same principles if we assume that the basis function map does not simply reflect the retinal image but receives instead a preprocessed version of the image. If the parietal cortex represents only the object that is attended, it is then possible to account for the interaction that has been recently reported between scene segmentation and neglect (Driver et al. 
1992; Halligan and Marshall 1994). We predict in particular that subjects with right parietal lesions will neglect the left side of the attended object - the one that has been segmented and selected - regardless of its position in space. This is indeed consistent with results reported from patients (Driver et al. 1992; Halligan and Marshall 1994).

If preprocessing of the image involves a normalization of the image for rotation - a form of mental rotation - then neglect of the left side of rotated objects can also be explained (Driver and Halligan 1991; Driver et al. 1994; Buxbaum et al. 1995). Similarly, if the basis function representation is the "screen". used for visual imagery, then the model can replicate the well-known inability of neglect patients to imagine the left side of a visual scene (Bisiach and Luzzatti 1978).

\section{Discussion}

The model of the parietal cortex presented here was originally developed by considering the response properties of parietal neurons and the computational constraints inherent in sensorimotor transformations. It was not designed to model neglect, so its ability to account for a wide range of deficits is additional evidence in favor of the basis function hypothesis.

As we have shown, our model captures three essential aspects of the neglect syndrome: (1) It reproduces the pattern of line crossing of parietal patients in line cancellation and line bisection experiments; (2) the deficit coexists in multiple frames of reference simultaneously; and (3) the model accounts for some of the object-based effects. These results rely in part on the existence of monotonic gradients along the retinal and eye position axis of the basis function map. As we have seen, the retinal gradient is supported by neurophysiological recordings in the parietal cortex (Andersen et al. 1990), but gradients for the postural signals remain to be demonstrated. The retinal gradient hypothesis is also at the heart of Kinsbourne's theory of hemineglect (Kinsbourne 1987) and some models of neglect dyslexia and line bisection are based on a similar idea (Mozer and Behrmann 1990; Mozer et al. in press).

Recent lesion experiments in monkeys suggest that, contrary to what was widely assumed, area 7 in the monkey may not be the homologue of the inferior parietal areas 39 and 40 in humans, the ones that are typically lesioned in the neglect syndrome (Watson et al. 1994). Instead, it would appear that the areas found in the superior temporal sulcus (STS) of the monkey cortex are the analogues to areas 30 and 40 . If this report is confirmed, then we predict that the responses of cells in the STS should have gain fields to integrate sensory and posture signals, as in the parietal cortex.

Our approach can account for many studies beyond the ones considered here by using similar computational principles. It can reproduce, in particular, the behavior of patients in line-bisection experiments (Halligan and Marshall 1989; 
Burnett-Stuart et al. 1991; Bisiach et al. 1994) and a variety of experiments dealing with frames of reference, whether in retinotopic, trunk-centered (Bisiach et al. 1985; Moscovitch and Behrmann 1994), environment-centered (Ladavas 1987; Farah et al. 1990) (i.e., with respect to gravity), or object-centered coordinates (Driver and Halligan 1991; Halligan and Marshall 1994; Husain 1995). It is also possible to account for the inability of parietal patients to imagine the contralesional side of a visual scene if visual imagery uses a basis function map as its "projection screen" (Bisiach and Luzzatti 1978). Finally, a model with a basis function map integrating sensory signals with vestibular inputs would also exhibit a temporary recovery after strong vestibular stimulation, as reported in humans following caloric stimulation of the inner ear. The mechanisms at play would be identical to the ones involved in the performance improvement on left targets in Karnath et al. (1993) experiments when subjects turn their head to the right (Figs. $1 \mathrm{~A}$ and $6 \mathrm{~A})$.

The results presented in this chapter have been obtained without using explicit representations of the various cartesian frames of reference (except for the retinotopy of the $\mathrm{BF}$ map). In fact, it is precisely because the lesion affected noncartesian representations that the model was able to reproduce these results. The lesion affects the functional space in which the basis functions are defined, which shares common dimensions with cartesian spaces, but cannot be reduced to them. Hence, a basis function map integrating retinal location and head position is retinotopic, but not solely retinotopic. Consequently, any attempt to determine the cartesian space in which hemineglect operates is bound to lead to inconclusive results in which cartesian frames of reference appear to be mixed.

It would be interesting to see if the basis function hypothesis could also account for sensorimotor adaptation, such as learning to reach accurately while wearing visual prisms. We predict that adaptation takes place in several frames of reference simultaneously, a prediction that is testable and would provide further support for the basis function framework.

Acknowledgements. This research was supported in part by a fellowship from the McDonnell-Pew Center for Cognitive Neuroscience to A.P. and grants from the Office of Naval Research and the Howard Hughes Medical Institute to T.J.S. We thank Daphne Bavelier for stimulating and inspiring discussions.

\section{References}

Andersen R, Essick G, Siegel R (1985) Encoding of spatial location by posterior parietal neurons. Science 230:456-458 


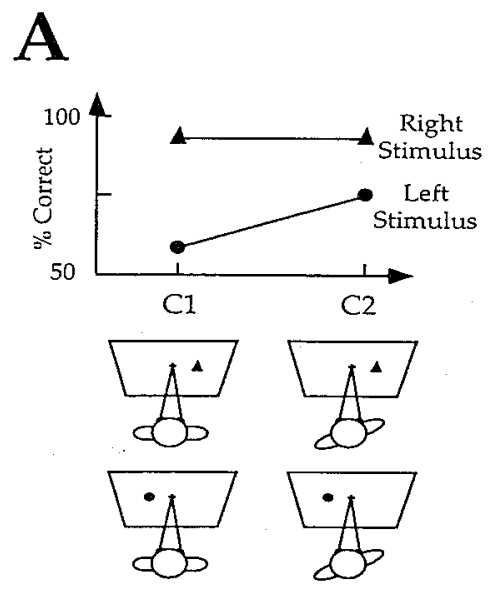

\section{B}

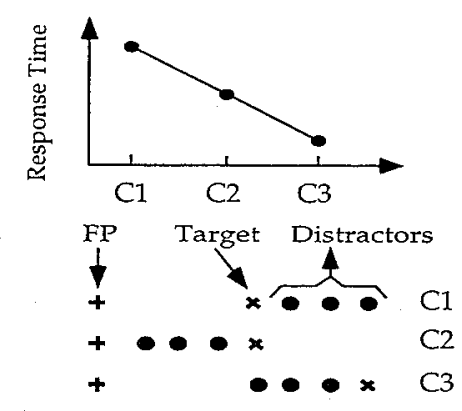

Fig. 1. A Percentage of correct identification in Karnath et al. experiment (1993). In condition $1(C l)$, subjects were seated with eyes, head, and trunk lined up whereas in condition 2 (C2) the trunk was rotated by $15^{\circ}$ to the left. The overall pattern of performance is not consistent with pure retinal or pure head-centered neglect and suggests a deficit affecting a mixture of these two frames of reference. B Response times for Arguin and Bub (1993) experiment for the three experimental conditions illustrated below the graph ( $F P$, fixation point). The decrease from condition $1(C 1)$ to condition $2(C 2)$ is consistent with object centered neglect, i.e., subjects are faster when the target is on the right of the distractors then when it is on the left, even though the retinal position of the target is the same. The further decrease in reaction time in condition $3(C 3)$ shows that the deficit is also retinotopic

As expected, subjects with right parietal lesions performed better on the right stimulus in the control condition (C1), a result consistent with both retinotopic and trunk-centered neglect. To distinguish between the two frames of reference, performance should be compared across conditions.

If the deficit is purely retinocentric, the results should be identical in both conditions since the retinotopic locations of the stimuli do not vary. On the other hand, if the deficit is purely trunk-centered, the performance on the left stimulus should improve when the head is turned right since the stimulus now appears further toward the right of the trunk-centered hemispace. Furthermore, performance on the right stimulus in the control condition should be the same as performance on the left stimulus in the rotated condition since they share the same trunk-centered position in both cases.

Neither of these hypotheses can fully account for the data. As expected from retinotopic neglect, subjects always performed better on the right stimulus in both conditions. However, performance on the left stimulus improved when the head was turned right (C2), though not sufficiently to match the level of performance on the right stimulus in the control condition (C1, Fig. 1A). Therefore, these results suggest a retinotopic neglect modulated by trunk-centered factors. 
In addition, Karnath et al. (1991) tested patients on a similar experiment in which subjects were asked to generate a saccade toward the target. The analysis of reaction time revealed the same type of results as the one found in the identification task, thereby demonstrating that the spatial deficit is, to a first approximation, independent of the task. Several other experiments have found that neglect affects a mixture of frames of reference in a variety of tasks (Ladavas 1987; Ladavas et al. 1989; Calvanio et al. 1987; Farah et al. 1990; Bisiach et al. 1985; Behrmann and Moscovitch 1994).

An experiment by Arguin and Bub (1993) suggests that neglect can be objectcentered as well. As shown in Fig. 1B, they found that reaction times were faster when a target (cross " $x$ " in Fig. 1B) appeared on the right of a set of distractors (C2) instead of on the left side (C1), even though the target is at the same retinotopic location in both conditions. Interestingly, moving the target further to the right led to even faster reaction times (C3), showing that hemineglect is not only object-centered but retinotopic as well in this task. Several other experiments have led to similar conclusions (Bisiach et al. 1979; Driver and Halligan 1991; Driver et al. 1994; Halligan and Marshall 1994; Husain 1995).

These results strongly support the existence of spatial representations using multiple frames of reference simultaneously shared by several behaviors. We recently developed a theory with these properties (Pouget and Sejnowski 1995, in press); we examine here whether a simulated lesion leads to a deficit similar to hemineglect. Our theory posits that parietal neurons compute basis functions (BFs) of sensory signals, such as visual or auditory inputs, and posture signals, such as eye or head position. The resulting representation, which we called a basis function map, can be used for performing nonlinear transformations of the sensory inputs - the type of transformations required for sensorimotor coordination.

The basis function hypothesis is briefly summarized in the first section of this chapter. In the second section, we describe the network architecture and the various methods used to assess the network performance in behavioral tests. In the third section, we compare the behavior of parietal patients with the network after a unilateral lesion of the basis function representation.

\section{Basis Function Representation}

The receptive field of most parietal cells is fixed on the retina, as for V1 neurons. The amplitude of their response to a light, however, is modulated by eye position (Andersen et al. 1985): typically, the response to a visual stimulus in the center of the receptive field increases monotonically as the eye moves along a particular direction in space, specific to each cell.

We have shown in a previous study that these response properties are consistent with the hypothesis that parietal neurons compute basis functions of their inputs 
A

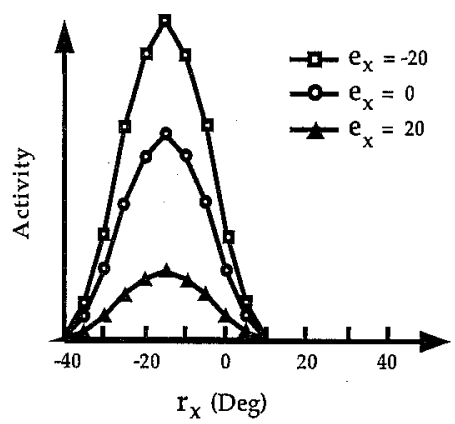

B

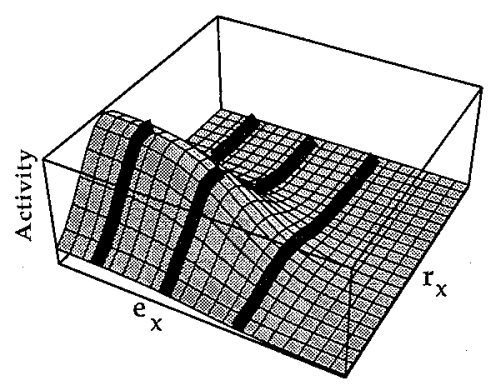

Fig. 2. A Idealization of a retinotopic visual receptive field of a typical parietal neuron for three different gaze angles $\left(e_{x}\right)$. Note that eye position modulates the amplitude of the response but does not affect the retinotopic position of the receptive field (adapted from Andersen et al. 1985). B 3D plot showing the response function of an idealized parietal neuron for all possible eye and retinotopic positions, $e_{x}$ and $r_{x}$. The plot in $A$ was obtained by mapping the visual receptive field of this idealized parietal neuron for three different eye positions as indicated by the bold lines

(Pouget and Sejnowski 1995, in press). Their response can be described as the product of a gaussian function of retinal location multiplied by a sigmoid function of eye position (Fig. 2B). Sets of both Gaussians and sigmoids are basis functions, and the set of all products of these two basis functions also forms basis functions over the joint space.

A set of basis functions has the property that any nonlinear function can be approximated by a linear combination of the basis functions. Therefore, basis functions reduce the computation of nonlinear mappings to a linear transformation - a simpler computation. Most sensorimotor transformations are nonlinear mappings of the sensory and posture signals into motor coordinates; hence, given a set of basis functions, the motor command can be obtained by a linear combination of these functions. Basis functions are precisely what parietal neurons appear to compute. This formalization entails that the parietal cortex recodes the sensory inputs in a format that facilitates the computation of motor commands. This perspective is consistent with the Goodale and Milner suggestion that the dorsal pathway mediates object manipulation (the "How" pathway), as opposed to simply localizing objects as Mishkin et al. previously suggested (the "Where" pathway) (Goodale and Milner 1990; Mishkin et al. 1983).

It is important to realize that not all models of parietal cells have the properties of simplifying the computation of nonlinear motor commands. For example, Goodman and Andersen (1990) as well as Mazzoni and Andersen (1995) have proposed that parietal cells simply add the retinal and eye position signals. The output of this linear model does not reduce the computation of motor commands to linear combinations because linear units cannot provide a basis set. By contrast, 
the hidden units of the Zipser and Andersen model (1988) have response properties closer to the basis function units, and the basis function hypothesis can be seen as a formalization of this previous model (for a detailed discussion see Pouget and Sejnowski, in press).

One particularly interesting property of basis functions is the fact that they represent the positions of objects in multiple frames of reference simultaneously. Thus, one can recover simultaneously the position of an object in retinotopic and head-centered coordinates from the response of a group of basis function units similar to the one shown in Fig. 2B (Pouget and Sejnowski 1995, in press). As shown in the next section, this property allows the same set of units to be used to perform multiple spatial transformations in parallel.

This approach can be readily extended to other sensory and posture signals and to other parts of the brain where similar gain modulations have been reported (Trotter et al. 1992; Field and Olson 1994; Boussaoud et al. 1993; Bremmer and Hoffmann 1993; Brotchie et al. 1995). When generalized to other posture signals such as vestibular inputs of head position, the resulting representation encodes simultaneously the retinal, head-centered, body-centered, and world centered coordinates of objects.

The study presented here explores the effects of a lesion in a spatial representation using basis functions. It is an attempt to bridge the gap between our current understanding of spatial representations at the neurophysiological and neuropschological levels.

\section{Model Organization}

The model contains two distinct parts: a network for performing sensorimotor transformations and a selection mechanism. The selection mechanism is used when there is more than one object present in the visual field at the same time.

\section{Network Architecture}

We implemented a network using basis function units in the intermediate layer to perform a transformation from a visual retinotopic map to two motor maps in head-centered and oculocentric coordinates respectively (Fig. 3). The input contains a retinotopic visual map analogous to the one found in the early stages of visual processing and a set of units encoding eye position, similar to the neurons found in the intralaminar nucleus of the thalamus (Schlag-Rey and Schlag 1984). These input units project to a set of intermediate units that contribute to both output transformations. Each intermediate unit computes a Gaussian of the retinal location of the object, $r_{x}$, multiplied by a sigmoid of eye position, $e_{x}$ : 


$$
o_{i j}=\frac{e^{-\frac{\left(r_{x}-r_{x i}\right)^{2}}{2 \sigma^{2}}}}{1+e^{-\beta\left(e_{x}-e_{x j}\right)}}
$$

We consider horizontal positions only because the vertical axis is irrelevant for hemineglect. These units are organized in two two-dimensional maps covering all possible combinations of retinal and eye position selectivities. The only difference between the two maps is the sign of the parameter $\beta$ which controls whether the units increase or decrease activity with eye position. $\beta$ was set to $8^{\circ}$ for one map and $-8^{\circ}$ for the other map. The indices $(i, j)$ refer to the position of the units on the maps. Each location is characterized by a position for the peak of the retinal receptive field, $r_{x i}$, and the midpoint of the sigmoid of eye position, $e_{x j}$. These quantities are systematically varied along the two dimensions of the maps in such a way that in the upper right corner $r_{x i}$ and $e_{x j}$ correspond to right retinal and right eye positions whereas in the lower left they correspond to left retinal and left eye positions.

As emphasized previously, this type of response function is consistent with the responses of single parietal neurons found in area $7 \mathrm{a}$. The resulting population of units forms basis function maps encoding the locations of objects in head-centered and retinotopic coordinates simultaneously.

A

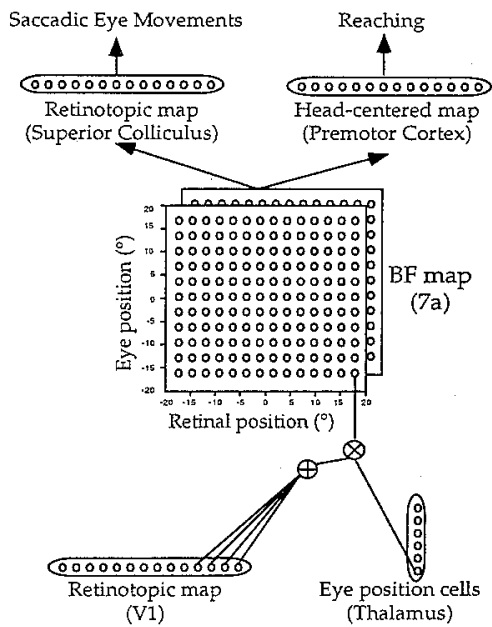

B

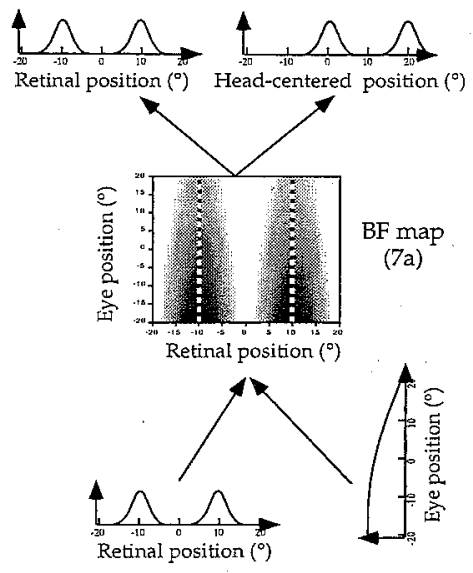

Fig. 3. A Network architecture. Each unit in the intermediate layers is a basis function unit with a gaussian retinal receptive field modulated by a sigmoid function of eye position. This type of modulation is characteristic of the response of parietal neurons. B Pattern of activity for two visual stimuli presented at $+10^{\circ}$ and $10^{\circ}$ on the retina with the eye pointing at $+10^{\circ}$ 
The activities of the units in the output maps are computed by a simple linear combination of the BF unit activities. Appropriate values of the weights were found by using linear regression to achieve the least mean square error.

This architecture mimics the pattern of projections of the parietal area $7 \mathrm{a}$, which projects to both the superior colliculus and the premotor cortex (via the ventral parietal area VIP; Andersen et al. 1990; Colby and Duhamel 1993), where neurons have retinotopic and head-centered visual receptive fields respectively. Figure 3B shows a typical pattern of activity in the network when two stimuli are presented simultaneously while the eye is fixated $10^{\circ}$ toward the right (only the BF map with positive $\beta=+8^{\circ}$ is shown).

\section{Hemispheric Biases and Lesion Model}

Neurophysiological data indicate that although the parietal cortices in both hemispheres contain neurons with all possible combinations of retinal and eye position selectivities, most cells tend to have their retinal receptive field on the contralateral side (Andersen et al. 1990). Whether a similar contralateral bias exist for the eye position in the parietal cortex remains to be determined although several authors have reported such bias for eye position selectivities in other parts of the brain (Schlag-Rey and Schlag 1984; Galletti and Battaglini 1989; van Opstal et al. 1995).

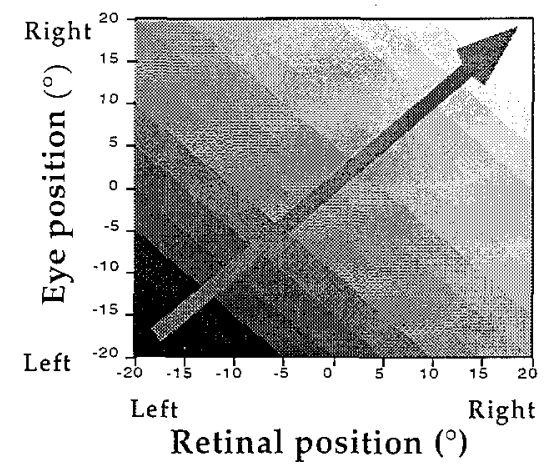

Left Map

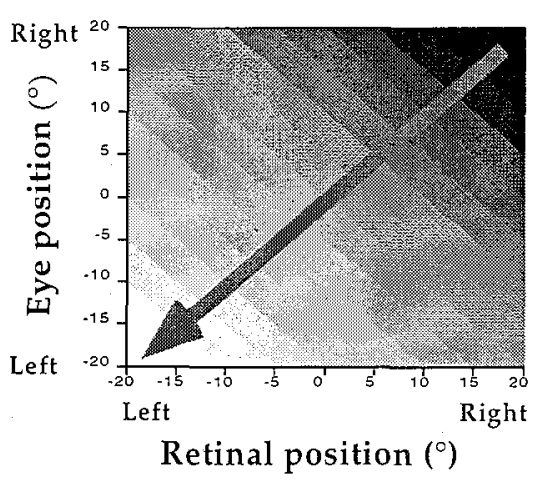

Right Map

Fig. 4. Neuronal gradients in left and right basis function maps for which the parameter $\beta$ is positive, i.e, the units increase activity with eye position. The right map contains more neurons for left retinal and left eye positions while the left map has the opposite gradient 
In the model, we divide the two BF maps into two sets of two maps, one set for each hemisphere (again the two maps in each hemisphere correspond to two possible values for the parameter $\beta$ ). Units are distributed across each hemisphere to create neuronal gradients. These neuronal gradients induce contralateral activity gradients such that there is more activity overall in the left maps than in the right maps when an object appears on the right of the retina and the eyes are turned to the right, with the opposite being true in the right maps.

Several types of neuronal gradients can lead to such activity gradients. The gradients we use for the simulations presented here affect only the maps with positive $\beta$, i.e, maps with units whose activity increases as the eyes turn right. In both the right and left map, the number of units for a given pair of $\left(r_{x i}, e_{x i}\right)$ increases for contralateral values of eye and retinal location as indicated in Fig. 4 which is consistent with the experimental observation that hemispheres overrepresent contralateral positions.

To model a right parietal lesion, we removed the right parietal maps and studied the network behavior with the left maps alone. The effect of the lesion is therefore to induce a neuronal gradient such that there is more activity in the network for right retinal and right eye positions.

We found that the exact profile of the neuronal gradient across the basis function maps did not matter as long as it induces a monotonically increasing activity gradient as objects are moved further to the right of the retina and the eyes fixate further to the right. The results presented in this chapter were obtained with linear neuronal gradients.

\section{Selection Model}

We adapted a selection mechanism from Burgess (1995) which itself was inspired by the visual search theory of Treisman and Gelade (1980) and the saliency map mechanism proposed by Koch and Ullman (1985). It was used to model the behavior of patients when presented with several stimuli simultaneously and it operates on what we call the saliency value associated with each stimulus.

The simultaneous presentation of stimuli induces multiple hills of activity in the network (see for instance the pattern of activity shown in Fig. 1B for two visual stimuli). We defined the stimulus saliency, $s_{i}$, as being the sum of the activities of all the basis function units whose receptive field is centered exactly on the retinal position of the stimulus (it is the sum of activities along the dotted line shown on the basis function map in Fig. 3B). The index $i$ varies from 1 to $n$, with $n$ as the number of stimuli in view at a given time. This method is mathematically equivalent to looking at the profile of activity in the superior colliculus output map and defining the saliency of the stimulus as the peak value of activity. Consequently, one need only consider the profiles of activity in the colliculus 
output map to determine the network behavior. Qualitatively similar values could also be obtained by looking at the profile of activation in the head-centered map.

At the first time step, the stimulus with the highest saliency is selected by winner-take-all, and its corresponding saliency is set to zero to implement inhibition of return. At the next time step, the second highest stimuli is selected, and inhibited, while the previously selected item is allowed to recover slowly. These operations are repeated for the duration of the trial. This procedure ensures that the most salient items are not selected twice in a row, but because of the recovery process, the stimuli with the highest saliencies might be selected again if displayed long enough.

This mechanism is such that the probability of selecting an item is proportional to two factors: the absolute saliency associated with the item and the saliency relative to the ones of competing items.

\section{Evaluating Network Performance}

We used this model to simulate several experiments in which patient performance was evaluated according to reaction time or percent of correct response.

In reaction time experiments, we assumed that processing involves two sequential steps: target selection and target processing. Target selection time was assumed to be proportional to the number of iterations, $n$, required by the selection network to select the stimulus using the mechanism described above. Each iteration was arbitrarily chosen to be $50 \mathrm{~ms}$ long. This duration matters only when more than one stimulus is present, so that distractors could delay the detection of the target by winning the competition.

The time (RT) for target processing (that is to say, target recognition, target naming, etc.) was assumed to be inversely proportional to stimulus saliency, $s_{i}$.

$$
R T=100+50 n+\frac{500}{1000 s_{i}} .
$$

We determined the percentage of correct responses to a stimulus to be a sigmoid of the stimulus saliency:

$$
p=\frac{0.5}{\left(1+\exp \left(-\left(s_{i}-s_{0}\right) / t\right)\right.}+0.5
$$

where $s_{o}$ and $t_{o}$ are constants.

This is a standard method in signal detection theory when assuming gaussian noise of equal variance for signal and noise (Green and Swets 1966). This is equivalent to assuming that the rate of correct detection (hit rate) is the integral of the probability distribution of the signal from the decision threshold to infinity. 
In line bisection experiments, subjects are asked to judge the midpoint of a line segment. Our network estimated the midpoint of a line, $\vec{m}$, by computing the center of mass of the activity induced by the line in the BF map.

$$
\vec{m}=\frac{\sum_{\text {all units }} a_{i} r_{x i}}{\sum_{\text {all units }} a_{i}}
$$

where $r_{x i}$ is the retinal position of the peak of the visual receptive field of unit $i$.

\section{Results}

All the results concern the lesioned model only, i.e., the model in which the right $\mathrm{BF}$ maps have been removed.

\section{Line Cancellation}

We first tested the network on the line cancellation test, a test in which patients are asked to cross out short line segments uniformly spread over a page. To simulate this test, we presented the display shown in Fig. 5A and ran the selection mechanism to determine which lines get selected by the network. As illustrated in Fig. 5A, the network crossed out only the lines located in the right half of the display, just as left neglect patients do in the same task (Heilman et al. 1985). The rightward gradient introduced by the lesion makes the right lines more salient than the left lines. As a result, the rightmost lines always win the competition, preventing the network from selecting the left lines.

We computed the probability that the line was crossed out as a function of its position in the display, where position is defined with respect to the frame of the display (Fig. 5A). We found that there is a sharp jump in the probability function such that lines on the right of this break have a probability near 1 of being selected whereas lines on the left of the break have a probability near zero (Fig. 5B).

The sharp jump in the probability of selection stands in contrast to the smooth and monotonic profile of the neuronal gradient. Whereas the sharp boundary in the pattern of line crossing may suggest that the model "sees" only one half of the display, the linear profile of the neuronal gradient shows that this is not the case. The sharp jump is mostly the consequence of the dynamics of the selection process: because right bars are associated with higher saliencies, they consistently win the competition to the detriment of left bars. Consequently, the network starts by selecting the bar the furthest on the right and due to inhibition of return moves its way toward the left. Eventually, however, previously inhibited items recover 
A

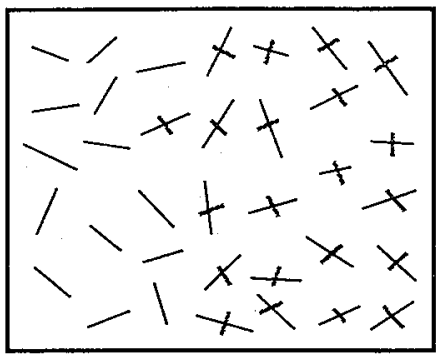

C
B

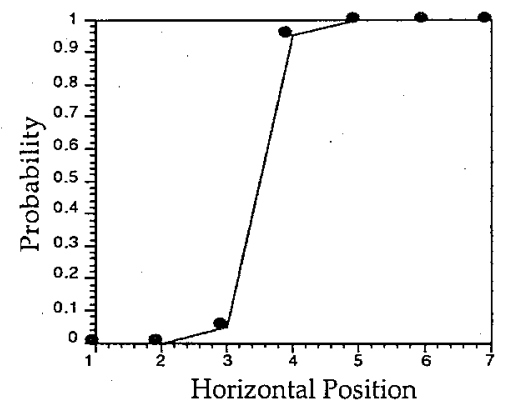

Fig. 5. A Network behavior in line cancellation task. As with right parietal patients, the network fails to cross out the line segments on the left of the page. B Probability of crossing a line as a function of its horizontal position in the display. The network behaves as if it had no representation of the left side of the display, i.e, as if the neuronal gradient introduced by the lesion were a step function. The gradient however is smooth, and the sudden change in behavior in the middle of the display is the result of the dynamics of the selection mechanism. C Network behavior in line bisection task. The midpoint is estimated too far to the right due to the overrepresentation of the right side of space

and win the competition again, preventing the network from selecting the leftmost bars. The point at which the network stops selecting bars toward the left depends on the exact recovery rate and the total number of items displayed.

The pattern of line crossing by the network is not the result of a deficiency in the selection mechanism. It is the result of a selection mechanism operating on a lesioned spatial representation. The network had trouble detecting stimuli on the left side of space not because it was unable to orient toward that side of space - it would orient to the left if only one stimulus were presented in the left hemifield but because the bias in the representation favored the rightmost bars in the competition.

\section{Line Bisection}

In the line bisection task, the network estimated the line midpoint to be slightly to the right of the actual midpoint (Fig. 5C) as reported in patients with left neglect (Heilman et al. 1985). In contrast, the performance of an intact network was perfect (not shown). 
The error was not because the lesioned network did not "see" the left side of the line. On the contrary, the whole line was represented in the lesioned network but due to the neuronal gradient, more neurons respond to the right side of the line than the left side. As a result, the center of mass calculation used to estimate the middle of the line leads to a rightward error.

Therefore, as assessed by the line cancellation and line bisection tests, a lesioned network exhibited a behavior consistent with the neglect syndrome observed in humans following unilateral parietal lesions.

\section{Mixture of Frames of Reference}

Next, we sought to determine the frame of reference of neglect in the model. Since Karnath et al. (1993) manipulated head position, we simulated their experiment by using a BF map integrating visual inputs with head position, rather than eye position. We show in Fig. $6 \mathrm{~B}$ the pattern of activity obtained in the retinotopic output layer of the network in the various experimental conditions. In both conditions, head straight ahead (dotted lines) or turned on the side (solid lines), the right stimulus is associated with more activity than the left stimulus. This is the consequence of the larger number of cells in the basis function map for
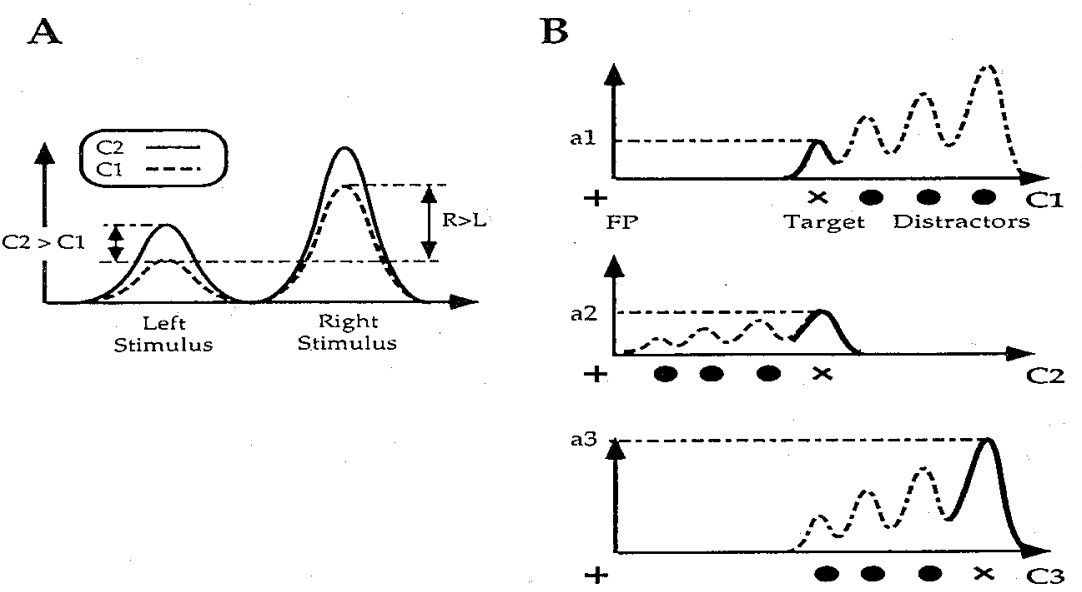

Fig. $6 \mathrm{~A}$, B. Activity patterns in the retinotopic output layer when simulating the experiments by A Karnath et al. (1993) and B Arguin and Bub (1993). A Performance on the left stimulus improves from condition $1(C l)$ to condition $2(C 2)$ because the stimulus saliency increases across conditions. $\mathbf{B}$ Reaction time between conditions 1 and 2 decreases due to the change in the relative saliency of the target with respect to the distractors, even though the absolute saliency of the target is the same in these two conditions $(a I=a 2)$. FP, Fixation point; $C 3$, condition 3 
rightward position. In addition, the activity for the left stimulus increases when the head is turned to the right. This effect is related to the larger number of cells in the basis function maps tuned to right head positions.

Since network performance is proportional to activity strength, the overall pattern of performance was found to be similar to what has been reported in human patients (Fig. 1A): the right stimulus was better processed than the left stimulus, and performance on the left stimulus increases when the head is rotated toward the right, although not sufficiently to match the peformance on the right stimulus in condition 1. Therefore, as in humans, neglect in the model is neither retinocentric nor trunk-centered alone but both at the same time.

Similar principles can be used to account for the behavior of patients in many other experiments dealing with frames of reference (Ladavas 1987; Ladavas et al. 1989; Calvanio et al. 1987; Farah et al. 1990; Bisiach et al. 1985; Behrmann and Moscovitch 1994).

\section{Object-Centered Effect}

The network reaction times in simulations of the Arguin and Bub (1993) experiments followed the same trends reported in human patients (Fig. 1B). Figure $6 \mathrm{~B}$ illustrates the patterns of activity in the retinotopic output layer of the network for the three conditions in those experiments. Although the absolute levels of activity associated with the target (solid lines) in conditions 1 and 2 are the same, the activity of the distractors (dotted lines) differed in the two conditions. In condition 1 , they had relatively higher activity and thereby strongly delayed the detection of the target by the selection mechanism. In condition 2 , the distractors were less active than the target and did not delay target processing as much as they did in condition 1 . The reaction time decreased even more in condition 3 because the absolute activity associated with the target was higher. Therefore, the network exhibited retinocentric and object-centered neglect, with the same pattern observed in parietal patients (Arguin and Bub 1993).

The object-centered effect might not have been expected since there was no explicit object-centered representation in the model. This result demonstrates that object-based neglect does not necessarily imply that an explicit object-based. representation has been lesioned in neglect patients. The form of neglect found in the Arguin and Bub (1993) experiment could be a consequence of relative neglect since the apparent object-based effect could be explained by the relative saliency of the subparts of the object.

Other results in neglect can be explained with the same principles if we assume that the basis function map does not simply reflect the retinal image but receives instead a preprocessed version of the image. If the parietal cortex represents only the object that is attended, it is then possible to account for the interaction that has been recently reported between scene segmentation and neglect (Driver et al. 
1992; Halligan and Marshall 1994). We predict in particular that subjects with right parietal lesions will neglect the left side of the attended object - the one that has been segmented and selected - regardless of its position in space. This is indeed consistent with results reported from patients (Driver et al. 1992; Halligan and Marshall 1994).

If preprocessing of the image involves a normalization of the image for rotation - a form of mental rotation - then neglect of the left side of rotated objects can also be explained (Driver and Halligan 1991; Driver et al. 1994; Buxbaum et al. 1995). Similarly, if the basis function representation is the "screen". used for visual imagery, then the model can replicate the well-known inability of neglect patients to imagine the left side of a visual scene (Bisiach and Luzzatti 1978).

\section{Discussion}

The model of the parietal cortex presented here was originally developed by considering the response properties of parietal neurons and the computational constraints inherent in sensorimotor transformations. It was not designed to model neglect, so its ability to account for a wide range of deficits is additional evidence in favor of the basis function hypothesis.

As we have shown, our model captures three essential aspects of the neglect syndrome: (1) It reproduces the pattern of line crossing of parietal patients in line cancellation and line bisection experiments; (2) the deficit coexists in multiple frames of reference simultaneously; and (3) the model accounts for some of the object-based effects. These results rely in part on the existence of monotonic gradients along the retinal and eye position axis of the basis function map. As we have seen, the retinal gradient is supported by neurophysiological recordings in the parietal cortex (Andersen et al. 1990), but gradients for the postural signals remain to be demonstrated. The retinal gradient hypothesis is also at the heart of Kinsbourne's theory of hemineglect (Kinsbourne 1987) and some models of neglect dyslexia and line bisection are based on a similar idea (Mozer and Behrmann 1990; Mozer et al. in press).

Recent lesion experiments in monkeys suggest that, contrary to what was widely assumed, area 7 in the monkey may not be the homologue of the inferior parietal areas 39 and 40 in humans, the ones that are typically lesioned in the neglect syndrome (Watson et al. 1994). Instead, it would appear that the areas found in the superior temporal sulcus (STS) of the monkey cortex are the analogues to areas 30 and 40. If this report is confirmed, then we predict that the responses of cells in the STS should have gain fields to integrate sensory and posture signals, as in the parietal cortex.

Our approach can account for many studies beyond the ones considered here by using similar computational principles. It can reproduce, in particular, the behavior of patients in line-bisection experiments (Halligan and Marshall 1989; 
Burnett-Stuart et al. 1991; Bisiach et al. 1994) and a variety of experiments dealing with frames of reference, whether in retinotopic, trunk-centered (Bisiach et al. 1985; Moscovitch and Behrmann 1994), environment-centered (Ladavas 1987; Farah et al. 1990) (i.e., with respect to gravity), or object-centered coordinates (Driver and Halligan 1991; Halligan and Marshall 1994; Husain 1995). It is also possible to account for the inability of parietal patients to imagine the contralesional side of a visual scene if visual imagery uses a basis function map as its "projection screen" (Bisiach and Luzzatti 1978). Finally, a model with a basis function map integrating sensory signals with vestibular inputs would also exhibit a temporary recovery after strong vestibular stimulation, as reported in humans following caloric stimulation of the inner ear. The mechanisms at play would be identical to the ones involved in the performance improvement on left targets in Karnath et al. (1993) experiments when subjects turn their head to the right (Figs. $1 \mathrm{~A}$ and $6 \mathrm{~A})$.

The results presented in this chapter have been obtained without using explicit representations of the various cartesian frames of reference (except for the retinotopy of the BF map). In fact, it is precisely because the lesion affected noncartesian representations that the model was able to reproduce these results. The lesion affects the functional space in which the basis functions are defined, which shares common dimensions with cartesian spaces, but cannot be reduced to them. Hence, a basis function map integrating retinal location and head position is retinotopic, but not solely retinotopic. Consequently, any attempt to determine the cartesian space in which hemineglect operates is bound to lead to inconclusive results in which cartesian frames of reference appear to be mixed.

It would be interesting to see if the basis function hypothesis could also account for sensorimotor adaptation, such as learning to reach accurately while wearing visual prisms. We predict that adaptation takes place in several frames of reference simultaneously, a prediction that is testable and would provide further support for the basis function framework.

Acknowledgements. This research was supported in part by a fellowship from the McDonnell-Pew Center for Cognitive Neuroscience to A.P. and grants from the Office of Naval Research and the Howard Hughes Medical Institute to T.J.S. We thank Daphne Bavelier for stimulating and inspiring discussions.

\section{References}

Andersen R, Essick G, Siegel R (1985) Encoding of spatial location by posterior parietal neurons. Science 230:456-458 
Andersen R, Asanuma C, Essick G, Siegel R (1990) Corticocortical connections of anatomically and physiologically defined subdivisions within the inferior parietal lobule. J Comp Neurol 296:65-113

Arguin M, Bub D (1993) Evidence for an independent stimulus-centered reference frame from a case of visual hemineglect. Cortex 29:349-357

Behrmann M, Moscovitch M (1994) Objects-centered neglect in patients with unilateral neglect: effects of left-right coordinates of objects. J Cogn Neurosci 6:151-155

Bisiach E, Luzzatti C (1978) Unilateral neglect of representational space. Cortex 14:129133

Bisiach E, Luzzatti C, Perani D (1979) Unilateral neglect, representational schema and conciousness. Brain 102:609-618

Bisiach E, Capitani E, Porta E (1985) Two basic properties of space representation in the brain: evidence from unilateral neglect. J Neurol Neurosurg Psychiatry 48:141-144

Bisiach E, Rusconi M, Peretti V, Vallar G (1994) Challenging current accounts of unilateral neglect. Neuropsychologia 32:1431-1434

Boussaoud D, Barth T, Wise S (1993) Effects of gaze on apparent visual responses of frontal cortex neurons. Exp Brain Res 93:423-434

Bremmer F, Hoffmann K (1993) Pursuit related activity in macaque visual cortical areas MST and LIP is modulated by eye position. Soc Neurosci Abstr: 1283

Brotchie P, Andersen R, Snyder L, Goodman S (1995) Head position signals used by parietal neurons to encode locations of visual stimuli. Nature 375:232-235

Burgess N (1995) A solvable connectionist model of immediate recall of ordered lists. In: Tesauro G, Touretzky D, Leen $T$ (eds) Advances in neural information processing Systems, vol 7. MIT Press, Cambridge

Burnett-Stuart G, Halligan P, Marshall J (1991) A newtonian model of perceptual distortion in visuo-spatial neglect. Neuroreport 2:255-257

Buxbaum LJ, Coslett HB, Montgomery MW, Farah MJ (1996) Mental rotation may underlie apparent object-based neglect. Neuropsychologia 34:112-126

Calvanio R, Petrone P, Levine D (1987) Left visual spatial neglect is both environmentcentered and body-centered. Neurology 37:1179-1181

Colby C, Duhamel J (1993) Ventral intraparietal area of the macaque: anatomic location and visual response properties. J Neurophysiol 69:902-914

Colby C, Duhamel J, Goldberg M (1995) Oculocentric spatial representation in parietal cortex. Cereb Cortex 5:470-481

Driver J, Halligan P (1991) Can visual neglect operate in object-centered coordinates? An affirmative single case study. Cogn Neuropsychol 8:475-496

Driver J, Baylis G, Rafal R (1992) Preserved figure-ground segregation and symmetry perception in visual neglect. Nature 360:73-75

Driver J, Baylis G, Goodrich S, Rafal R (1994) Axis-based neglect of visual shapes. Neuropsychologia 32:1353-1365

Farah M, Brunn J, Wong A, Wallace M, Carpenter P (1990) Frames of reference for allocating attention to space: evidence from the neglect syndrome. Neuropsychologia 28:335-47

Field $P$, Olson C (1994) Spatial analysis of somatosensory and visual stimuli by single neurons in macaque area 7B. Soc Neurosci Abstr 20:317.12

Fogassi L, Gallese V, di Pellegrino G, Fadiga L, Gentilucci M, Luppino G, Matelli M, Pedotti A, Rizzolatti G (1992) Space coding by premotor cortex. Exp Brain Res 89:686690 
Galletti C, Battaglini P (1989) Gaze-dependent visual neurons in area V3a of monkey prestriate cortex. J Neurosci 9:1112-1125

Goldberg M, Colby C, Duhamel J (1990) Representation of visuomotor space in the parietal lobe of the monkey. Cold Spring Harb Symp Quant Biol 55:729-739

Goodale M, Milner A (1990) Separate visual pathways for perception and action. Trends Neurosci 15:20-25

Goodman S, Andersen R (1990) Algorithm programmed by a neural model for coordinate transformation. International Joint Conference on Neural Networks, San Diego

Graziano M, Yap G, Gross C (1994) Coding of visual space by premotor neurons. Science 266:1054-1057

Green D, Swets J (1966) Signal detection theory and psychophysics. Wiley, New York

Halligan P, Marshall J (1989) Line bisection in visuo-spatial neglect: disproof of a conjecture. Cortex 25:517-521

Halligan P, Marshall J (1994) Figural perception and parsing in visuospatial neglect. Neuroreport 5:537-539

Heilman K, Watson R, Valenstein E (1985) Neglect and related disorders. In: Heilman K, Valenstein E (eds) Clinical neuropsychology. Oxford University Press, New York, pp 243-294

Husain M (1995) Is visual neglect body-centric? J Neurol Neurosurg Psychiatry 58:262263

Karnath H-O, Schenkel P, Fischer B (1991) Trunk orientation as the determining factor of the 'contralateral' deficit in the neglect syndrome and as the physical anchor of the internal representation of body orientation in space. Brain 114:1997-2014

Karnath H-O, Christ K, Hartje W (1993) Decrease of contralateral neglect by neck muscle vibration and spatial orientation of trunk midline. Brain 116:383-396

Kinsbourne M (1987) Mechanisms of unilateral neglect. In: Jeannerod $M$ (ed) Neurophysiological and Neuropsychological aspects of spatial neglect. North-Holland, pp 69-86

Koch C, Ullman S (1985) Shifts in selective visual attention: towards the underlying neural circuitry. Human Neurobiol 4:219-227

Ladavas $E$ (1987) Is the hemispatial deficit produced by right parietal lobe damage associated with retinal or gravitational coordinates? Brain 110:167-180

Ladavas E, Pesce M, Provinciali L (1989) Unilateral attention deficits and hemispheric asymmetries in the control of visual attention. Neuropsychologia 27:353-366

Mazzoni P, Andersen R (1995) Gaze coding in the posterior parietal cortex. In: Arbib M (ed) The handbook of brain theory and neural networks. MIT Press, Cambridge, pp 423426

Mishkin M, Ungerleider L, Macko K (1983) Object vision and spatial vision: two cortical pathways. Trends Neurosci Oct: 414-417

Moscovitch M, Behrmann M (1994) Coding of spatial information in the somatosensory system: evidence from patients with neglect following parietal lobe damage. J Cogn Neurosci 6:151-155

Mozer M, Behrmann $M$ (1990) On the interaction of selective attention and lexical knowledge: a connectionist account of neglect dyslexia. J Cogn Neurosci 2:96-123

Mozer M, Halligan P, Marshall $J$ (in press) The end of the line for a brain-damaged model of hemispatial neglect. J Cogn Neurosci

Pouget A, Sejnowski T (1995) Spatial representations in the parietal cortex may use basis functions. In: Tesauro G, Touretzky $D$, Leen $T$ (eds) Advances in neural information processing systems, vol 7. MIT Press, Cambridge 
Pouget A, Sejnowski T (in press) Spatial transformations in the parietal cortex using basis functions. J Cogn Neurosci

Schlag-Rey M, Schlag J (1984) Visuomotor functions of central thalamus in monkey. I. unit activity related to spontaneous eye movements. J Neurophysiol 51:1149-1174

Stein J (1992) The representation of egocentric space in the posterior parietal cortex. Behav Brain Sci 15:691-700

Treisman A, Gelade G (1980) A feature integration theory of attention. Cogn Psych 12:97136

Trotter Y, Celebrini S, Stricanne B, Thorpe S, Imbert M (1992) Modulation of neural stereoscopic processing in primate area V1 by the viewing distance. Science 257:12791281

van Opstal A, Hepp K, Suzuki Y, Henn V (1995) Influence of eye position on activity in monkey superior colliculus. J Neurophysiol 74:1593-1610

Watson R, Valenstein E, Day A, Heilman $\mathbb{K}$ (1994) Posterior neocortical systems subserving awareness and neglect. Arch Neurol 51:1014-1021

Zipser D, Andersen R (1988) A back-propagation programmed network that stimulates reponse properties of a subset of posterior parietal neurons. Nature 331:679-684 